\title{
Crack and Electrical Resistance Behaviors of Carbon Nanotube-Based Polymer Composites under Mixed-Mode I/II Loading
}

\author{
Tomo Takeda*1, Yasuhide Shindo, Fumitsugu Naraoka*2, Yu Kuronuma*3 and Fumio Narita \\ Department of Materials Processing, Graduate School of Engineering, Tohoku University, Sendai 980-8579, Japan
}

This paper studies the crack and electrical resistance behaviors of carbon nanotube (CNT)-based polymer composites under mixed-mode I/II loading by theoretical and experimental approaches. Mixed-mode fracture tests were performed on single-edge cracked plate specimens of the nanocomposites, and the electrical resistance of the specimens was measured. Also, the crack growth direction was predicted by a fracture mechanics approach. In addition, an analytical model based on the electrical conduction mechanism of CNT-based composites was developed to describe the electrical resistance change as a result of inclined crack growth. The predictions on the crack growth direction and the crack induced resistance change were compared with the experimental data, and good agreements were found. [doi:10.2320/matertrans.M2013080]

(Received March 1, 2013; Accepted April 15, 2013; Published May 24, 2013)

Keywords: mesomechanics, material testing, modeling, carbon nanotubes, polymer-matrix composites, electrical properties, fracture, selfsensing

\section{Introduction}

Carbon nanotubes (CNTs) have received a significant amount of attention within the scientific and engineering communities due to their extraordinary properties compared to those of conventional materials. ${ }^{\text {) }}$ In addition, CNTs exhibit low density and high aspect ratio (length/diameter). Due to this unique combination of the characteristics, CNTs have emerged as suitable candidates for use as nanofillers in polymeric materials to yield next generation multifunctional composite systems. ${ }^{2,3)}$ Such nanocomposites have the potential to find increasingly wide-ranging applications. ${ }^{4,5}$ ) Over the years, many efforts have been made to understand the physical and mechanical characteristics of CNT-based polymer composites (e.g., review articles, ${ }^{6-8)}$ our recent works $\left.{ }^{9,10)}\right)$.

Structural health monitoring is a procedure for nondestructively identifying the current state of a component or system and detecting damage or degradation that could ultimately lead to a system failure. Many researchers have attempted to use CNT-based polymer composites as strain or damage sensors. ${ }^{11}$ ) The principle behind this is that the deformation or the damage initiation and evolution can lead to changes in the electrical resistance of CNT-based polymer composites. These electrical resistance changes provide the possibility of self-diagnostics and real-time health monitoring.

The possible presence of cracks is one of the potential problems that compromise the integrity of materials and engineering structures. With regard to the sensing ability of cracked CNT-based polymer composites, our research group $^{12)}$ investigated the crack and electrical resistance behaviors of multi-walled nanotube (MWNT)/polycarbonate composites under Mode I loading condition by using theoretical and experimental approaches. Recently, using MWNTs, Cardoso et al. ${ }^{13)}$ studied the damage initiation and

\footnotetext{
${ }^{* 1}$ Corresponding author, E-mail: takeda-t@material.tohoku.ac.jp

${ }^{* 2}$ Research Student, Tohoku University

${ }^{* 3}$ Graduate Student, Tohoku University
}

evolution within the process zone ahead of the crack tip of epoxy particulate composites under Mode I loading.

In practical situations, materials and engineering structures are subjected to mixed-mode loading, and deflected or inclined cracks can be encountered. For the realization of multifunctional composite systems based on CNTs, a thorough and comprehensive research on the mixed-mode crack behavior and associated electrical response of CNTbased polymer composites is essentially needed. In this paper, we investigate both theoretically and experimentally the crack and electrical resistance behaviors of CNT-based polymer composites subjected to mixed-mode I/II loading. Fracture tests were conducted on single-edge cracked plate specimens of the nanocomposites under mixed-mode I/II loading conditions. During the tests, the electrical resistance of the specimens was measured. Also, the direction of the mixedmode crack growth was predicted by a fracture mechanics approach. In addition, an analytical model for describing the electrical resistance change due to inclined crack growth was developed based on the electrical conduction mechanism of CNT-based composites, and the crack sensing ability of the nanocomposites was characterized.

\section{Experimental Procedure}

\subsection{Material and specimen preparation}

Nanocomposites consisting of MWNTs $(10-40 \mathrm{~nm}$ diameter, 5-20 $\mu \mathrm{m}$ length) and a polycarbonate matrix were considered in this study. The MWNT/polycarbonate composite samples were manufactured by a commercial supplier (Takiron Co., Ltd., Japan). The nanocomposite samples contained MWNT weight fraction of 2.5 mass $\%$.

Single-edge cracked specimens were used for experiments. The test specimens with a width $W=18 \mathrm{~mm}$ and a length $L=180 \mathrm{~mm}$ were machined from the sample plates. The as-received thickness of the plates was about $2.7 \mathrm{~mm}$. The geometrical details of the specimens are given in Fig. 1. A notch of width $1 \mathrm{~mm}$ and length $9 \mathrm{~mm}$ was machined at one edge of the specimen, and a sharp crack was introduced by tapping a razor blade into the preformed notch. Before 


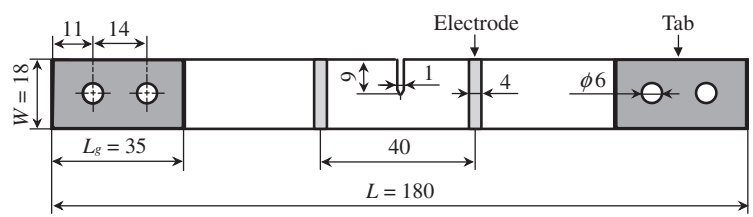

unit: $\mathrm{mm}$

Fig. 1 Geometry and dimensions of single-edge cracked plate specimen.

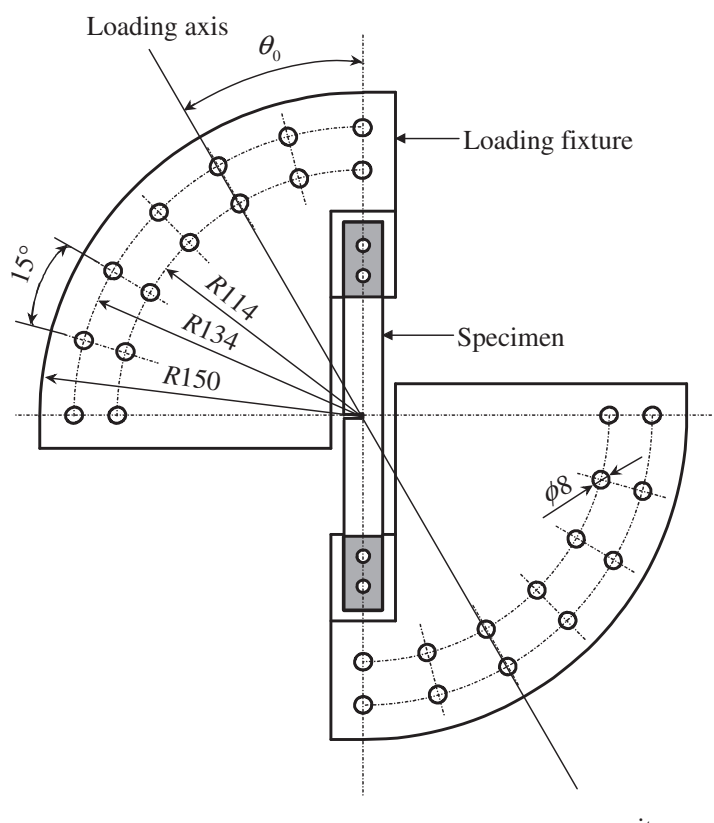

Fig. 2 Mixed-mode fracture test fixture.

testing, the notch tip was imaged using a digital microscope in order to determine the initial crack length $a_{0} \approx 9.3 \mathrm{~mm}$. For electrical resistance measurement, conductive silver paint was attached on one surface of the specimens as electrodes. The electrode width and the inter-electrode distance were 4 and $40 \mathrm{~mm}$, respectively. Non-conducting tabs with a length $L_{g}=35 \mathrm{~mm}$ were bonded to the specimen ends to electrically isolate the specimen from the metallic test fixture.

\subsection{Fracture testing and electrical measurements}

Fracture tests were carried out at ambient temperature in a servo-hydraulic testing machine. The single-edge cracked specimens were loaded under displacement control (0.6 $\mathrm{mm} / \mathrm{min}$ ). Mixed-mode I/II loading was applied using a loading fixture, shown in Fig. 2. By changing the loading angle $\theta_{0}$, the loading axes on the specimen can be changed and different mixed-mode conditions at the crack tip can be obtained. The specimens were tested at two selected loading angles $\left(0\right.$ and $\left.30^{\circ}\right)$. Load $P$ and displacement $\delta$ were recorded by the testing machine load cell and transducer, respectively. During the fracture tests, the region around the crack tip was observed using a digital microscope, and the projected length $A$ of the inclined crack shown in Fig. 3 was obtained. Specimen resistance $R$ was also measured with a voltage-current meter (R8340A, ADVANTEST Corporation, Japan). In practice, two specimens were tested for each condition.

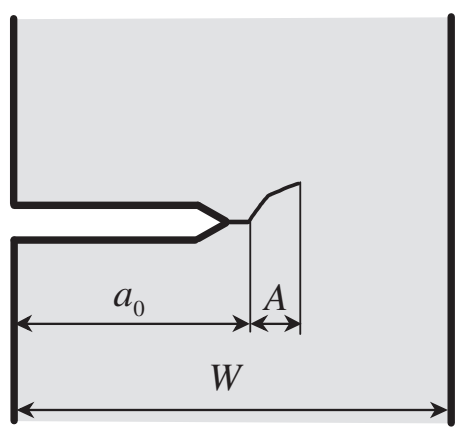

Fig. 3 Crack region of single-edge cracked plate specimen.

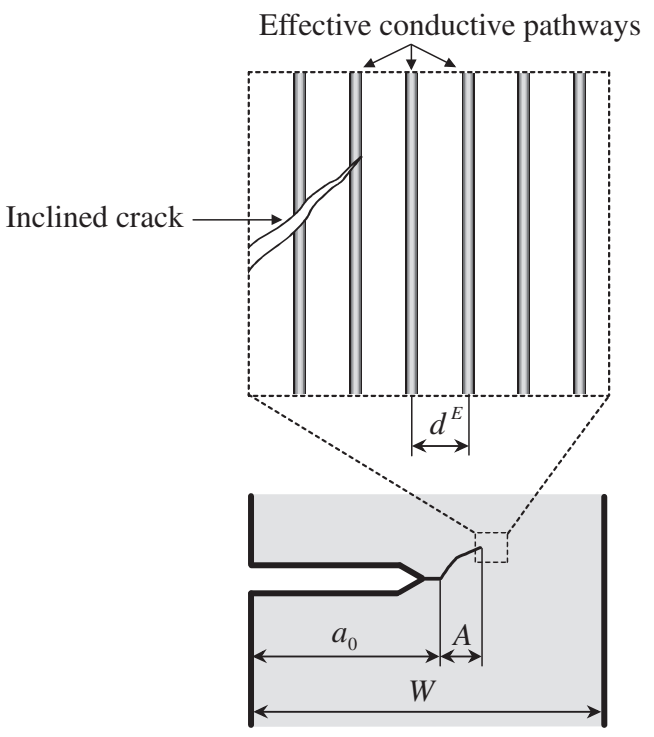

Fig. 4 Effective conductive pathways in the single-edge cracked specimen with inclined crack.

\section{Theoretical Procedure}

\subsection{Prediction of the electrical resistance change due to inclined crack growth}

It is well known that CNTs are able to form electrically conductive networks in polymers. There are some continuous conductive pathways in nanotube networks, which carry current and play a fundamental role in the electrical transport process in the composite system. ${ }^{14)}$ Based on Shindo et al. ${ }^{12)}$ the pathways were modeled as straight and parallel conductive wires (effective conductive pathways). The concept of the effective conductive pathways in the singleedge cracked specimen of CNT-based polymer composites with inclined crack is illustrated in Fig. 4. It was assumed that the distance between the effective conductive pathways $d^{E}$ was constant and all effective conductive pathways had the same resistance $R^{E}$. The superscript $E$ indicates the effective conductive pathway. The number of the effective conductive pathways in the initial ligament region $n_{0}^{E}$ can be expressed in terms of the specimen width $W$ and the initial crack length $a_{0}$ as

$$
n_{0}^{E}=\left\lfloor\frac{W-a_{0}}{d^{E}}\right\rfloor,
$$


where \lfloor\rfloor denotes the floor function, that is, the largest integer less than or equal to a given real number. The initial electrical resistance of the composite specimen $R_{0}$ is given by

$$
R_{0}=\frac{R^{E}}{n_{0}^{E}} .
$$

The propagation of the inclined crack breaks conductive pathways in the specimen, and the number of the effective conductive pathways in the ligament region after inclined crack propagation $n_{\mathrm{p}}^{E}$ can be obtained as

$$
n_{\mathrm{p}}^{E}=\left\lfloor\frac{W-a_{0}-A}{d^{E}}\right\rfloor .
$$

The specimen resistance after inclined crack propagation $R_{\mathrm{p}}$ is given by

$$
R_{\mathrm{p}}=\frac{R^{E}}{n_{\mathrm{p}}^{E}} .
$$

Thus the change in the specimen resistance due to inclined crack propagation $\Delta R_{\mathrm{p}}=R_{\mathrm{p}}-R_{0}$ normalized by the initial resistance $R_{0}$ can be approximately written as

$$
\frac{\Delta R_{\mathrm{p}}}{R_{0}}=\frac{A}{W-a_{0}-A} .
$$

\subsection{Prediction of the crack growth angle}

Based on the fracture mechanics approach, the initial direction of the mixed-mode crack growth was predicted. Figure 5 shows the initial crack growth angle $\theta_{\mathrm{p}}$ in which the polar coordinates $(r, \theta)$ at the initial crack tip are used. The minimum strain energy density criterion ${ }^{15)}$ was used to determine the crack growth direction. In the case of mixedmode I/II loading, the strain energy density factor $S$ is given by

$$
S=\alpha_{11} K_{\mathrm{I}}^{2}+2 \alpha_{12} K_{\mathrm{I}} K_{\mathrm{II}}+\alpha_{22} K_{\mathrm{II}}^{2},
$$

where $K_{\mathrm{I}}$ and $K_{\mathrm{II}}$ are the Mode I and Mode II stress intensity factors, respectively. The coefficients $\alpha_{i j}(i, j=1,2)$ are given by

$$
\begin{aligned}
& \alpha_{11}=\frac{1}{16 G}\{(1+\cos \theta)(\kappa-\cos \theta)\}, \\
& \alpha_{12}=\frac{1}{16 G} \sin \theta\{2 \cos \theta-(\kappa-1)\}, \\
& \alpha_{22}=\frac{1}{16 G}\{(\kappa+1)(1-\cos \theta)+(1+\cos \theta)(3 \cos \theta-1)\},
\end{aligned}
$$

where $G$ is the shear modulus. For the present problem, the parameter $\kappa$ is as follows

$$
\kappa=\frac{3-v}{1+v},
$$

where $v$ is the Poisson's ratio. In the minimum strain energy density criterion, it is assumed that the crack growth angle $\theta_{\mathrm{p}}$ coincides with the value of $\theta$ which yields the relative minimum value of $S$, i.e.,

$$
\left(\frac{\partial S}{\partial \theta}\right)_{\theta=\theta_{\mathrm{p}}}=0,\left(\frac{\partial^{2} S}{\partial \theta^{2}}\right)_{\theta=\theta_{\mathrm{p}}}>0 .
$$

In order to determine the energy density factor $S$, we carried out a linear elastic finite element analysis for the

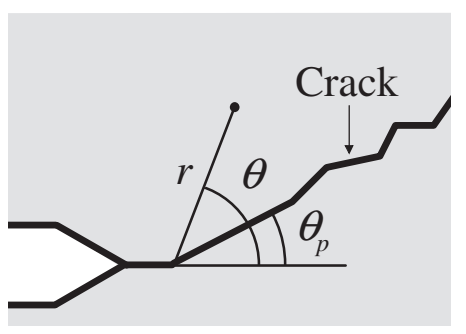

Fig. 5 Initial crack growth angle with the polar coordinates. (a)

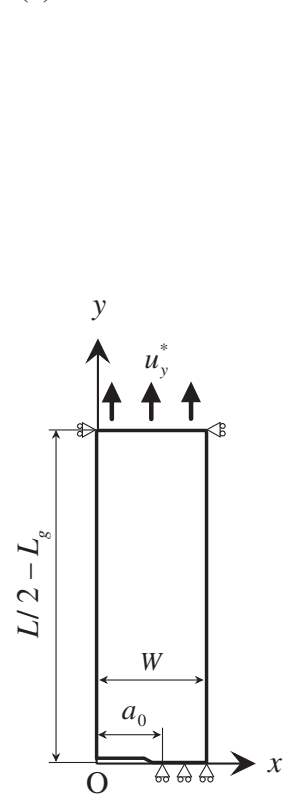

(b)

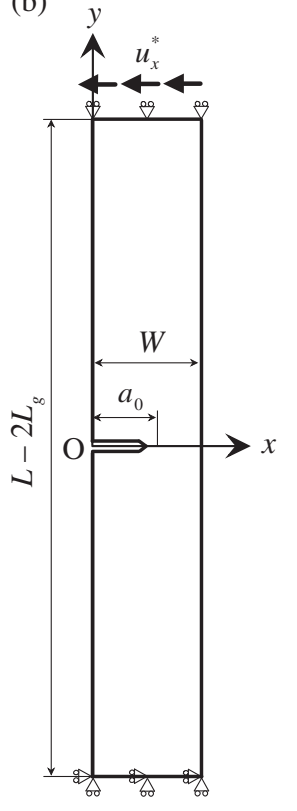

Fig. 6 Boundary conditions (a) for Mode I and (b) for Mode II loadings.

single-edge cracked specimens under both Mode I and Mode II loading conditions, and calculated the $J$-integrals. An accurate strain energy density factor can be obtained when the $J$-integral method is used since the accuracy of the $J$-integral method is insensitive to mesh refinement at the crack tip. ${ }^{16)}$ The commercial finite element code ANSYS was used for the analysis. The elastic material properties are: Young's modulus $E=2.56 \mathrm{GPa}$ and Poisson's ratio $v=$ $0.39 .{ }^{17)}$ Finite element solutions were obtained using a twodimensional plane stress model. In the model, the tab sections were not included. For Mode I loading, due to symmetry only a half of the specimen was considered. On the other hand, for Mode II loading, the finite element model treated the entire specimen. The boundary conditions for the finite element analysis are shown in Fig. 6, where O-xyz is the Cartesian coordinate system. The crack surface was traction free. Mode I and Mode II loadings were simulated by applying the constant prescribed displacements in the $y$ and $x$-directions $\left(u_{y}^{*}, u_{x}^{*}\right)$ at $y=L / 2-L_{g}(0 \leq x \leq W)$, respectively. The total nodal forces at $y=L / 2-L_{g}$ $(0 \leq x \leq W)$ for $u_{y}^{*}$ and $u_{x}^{*}$ correspond to the Mode I and Mode II components of the experimental load $\left(P \cos \theta_{0}\right.$, $\left.P \sin \theta_{0}\right)$, respectively. For the plane stress condition, the Mode I and Mode II stress intensity factors, $K_{\mathrm{I}}$ and $K_{\mathrm{II}}$, can be related to the $J$-integrals as follows: 


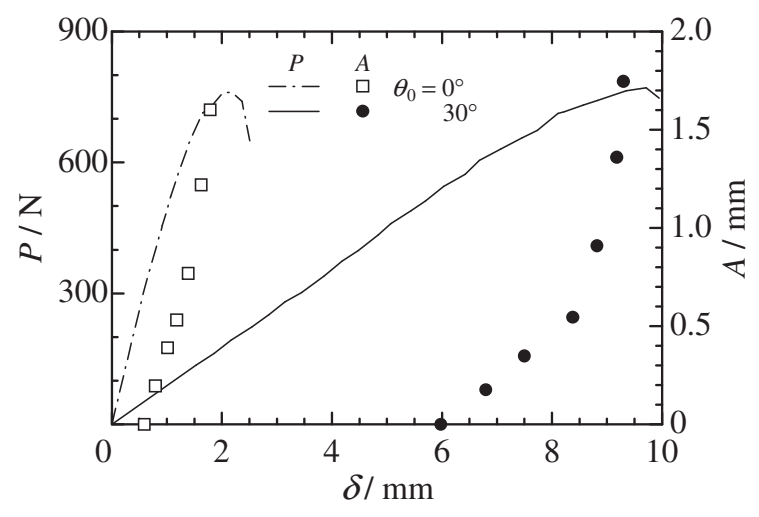

Fig. 7 Load and projected crack length versus displacement.

Table 1 Experimental and predicted results of the crack growth angles.

\begin{tabular}{ccc}
\hline & \multicolumn{2}{c}{$\theta_{\mathrm{p}}$} \\
\cline { 2 - 3 }$\theta_{0}$ & Experimental & $\begin{array}{c}\text { Minimum strain energy } \\
\text { density criterion }\end{array}$ \\
\hline $0^{\circ}$ & $0^{\circ}$ & $0^{\circ}$ \\
\hline $30^{\circ}$ & $31^{\circ}$ & $27^{\circ}$ \\
\hline
\end{tabular}

$$
K_{\mathrm{I}}^{2}=E J_{\mathrm{I}}, K_{\mathrm{II}}^{2}=E J_{\mathrm{II}},
$$

where $J_{\mathrm{I}}$ and $J_{\mathrm{II}}$ are the Mode I and Mode II $J$-integrals, respectively. The $J$-integral is given by

$$
\begin{aligned}
J= & \int_{\Gamma_{0}}\left\{w n_{x}-\left(\sigma_{x x} \frac{\partial u_{x}}{\partial x}+\sigma_{x y} \frac{\partial u_{y}}{\partial x}\right) n_{x}\right. \\
& \left.-\left(\sigma_{x y} \frac{\partial u_{x}}{\partial x}+\sigma_{y y} \frac{\partial u_{y}}{\partial x}\right) n_{y}\right\} \mathrm{d} \Gamma,
\end{aligned}
$$

where $\Gamma_{0}$ is a contour enclosing a crack tip, $w$ is the strain energy density, $\sigma_{x x}, \sigma_{y y}, \sigma_{x y}$ are the components of the stress tensor, $u_{x}, u_{y}$ are the components of the displacement vector, and $n_{x}, n_{y}$ are the components of the outer unit normal vector to $\Gamma_{0}$. Equation (11) was implemented numerically. Note that the present finite element analysis focuses only on the prediction of the crack growth direction in the single-edge cracked specimen of CNT-based polymer composites under mixed-mode I/II loading and the criterion for fracture will not be discussed in this paper.

\section{Results and Discussion}

Figure 7 shows the experimental results of the load $P$ and the projected crack length $A$ as a function of displacement $\delta$. The load-displacement curves are initially linear and then become nonlinear. The specimens exhibit stable crack growth, and in the case of loading angle $\theta_{0}=30^{\circ}$, the crack initiation is slow. Table 1 gives the experimental and predicted crack growth angles $\theta_{\mathrm{p}}$. The crack growth angles for both the front and back surfaces of the fractured specimens were measured based on the photographs and average values were taken. The crack propagates straight for $\theta_{0}=0^{\circ}$. On the other hand, for $\theta_{0}=30^{\circ}$, straight crack propagation does not occur and the inclined crack is observed. The predictions from the minimum strain energy density criterion are in good agreement with the experimental data.

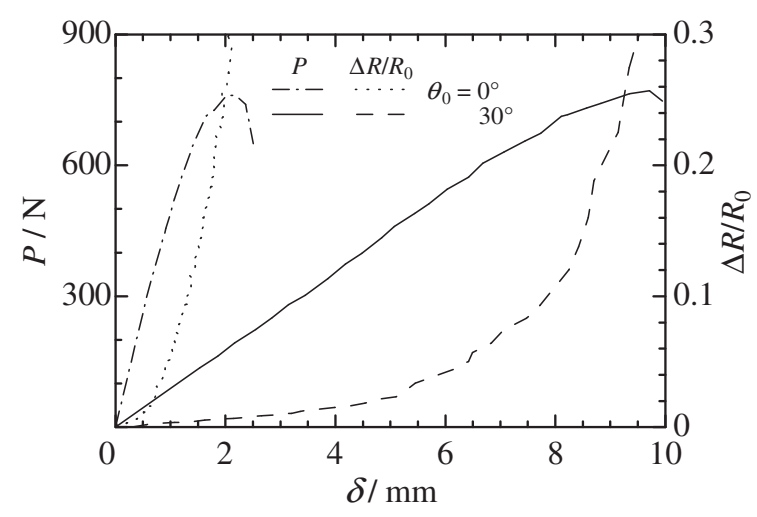

Fig. 8 Load and normalized resistance change versus displacement.

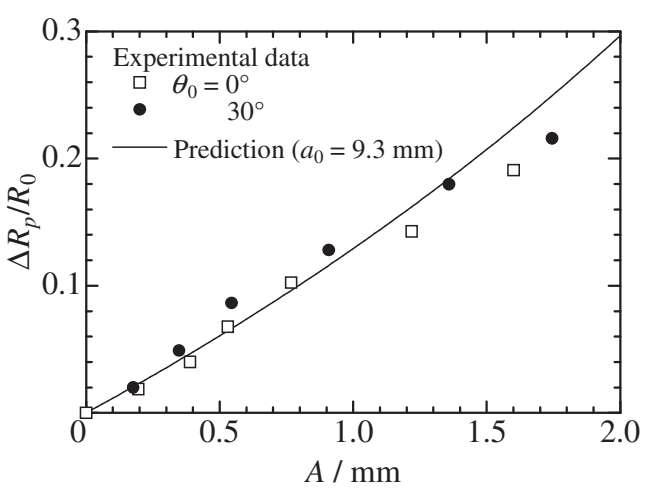

Fig. 9 Normalized resistance change due to crack propagation versus projected crack length.

The experimental data of the load $P$ and the normalized resistance change $\Delta R / R_{0}=\left(R-R_{0}\right) / R_{0}$ are plotted in Fig. 8 as a function of displacement $\delta$. A period of small resistance changes due to specimen deformation can be seen. But significant resistance changes occur after the onset of crack propagation (see also Fig. 7). In addition, the trends in the resistance change and projected crack length are similar and, therefore, it seems that the significant resistance changes are dominated by crack propagation. Figure 9 shows the relationship between the normalized resistance change due to crack propagation $\Delta R_{\mathrm{p}} / R_{0}=\left(\Delta R-\Delta R^{\prime}\right) / R_{0}$ and the projected crack length $A$ for the measured data, where $\Delta R^{\prime} / R_{0}$ is the normalized resistance change at the onset of crack propagation. The analytical prediction for $a_{0}=9.3 \mathrm{~mm}$ is also presented. The results indicate that the crack induced resistance change of CNT-based polymer composites does not depend on the crack growth direction. There is a reasonable agreement between the experimental and predicted results, and thus the present simple analytical model might be useful for predicting the electrical resistance response of cracked CNT-based polymer composites.

\section{Conclusions}

In this paper, the crack and electrical resistance responses of MWNT/polycarbonate composites under mixed-mode I/II loading were studied. Under mixed-mode I/II loading, an inclined crack was initiated, and the electrical resistance method was successfully applied to the monitoring of the 
mixed-mode crack behavior in the nanocomposites. An analytical model based on the electrical conduction mechanism of CNT-based composites was also developed for predicting the resistance change due to inclined crack growth, and the predicted results are in accordance with the experimental results. The present simple analytical model provides a fundamental insight into how the CNT networks interact with the inclined crack in nanocomposites. The crack sensing capability of CNT-based composites under mixedmode I/II loading enhances the potential of the use for the health monitoring of composite materials and various engineering structures.

\section{Acknowledgements}

The authors thank Takiron Co., Ltd. for providing the materials used in this study.

\section{REFERENCES}

1) R. H. Baughman, A. A. Zakhidov and W. A. de Heer: Science 297 (2002) 787-792.

2) T.-W. Chou, L. Gao, E. T. Thostenson, Z. Zhang and J.-H. Byun: Compos. Sci. Technol. 70 (2010) 1-19.
3) J. M. Wernik and S. A. Meguid: ASME Appl. Mech. Rev. 63 (2010) 050801.

4) J. Njuguna and K. Pielichowski: Adv. Eng. Mater. 5 (2003) 769-778.

5) A. Oliver, J. Bult, Q. V. Le, A. L. Mbaruku and J. Schwartz: Nanotechnology 19 (2008) 505702.

6) E. T. Thostenson, C. Li and T.-W. Chou: Compos. Sci. Technol. 65 (2005) 491-516.

7) K.-t. Lau, C. Gu and D. Hui: Compos. Part B 37 (2006) 425-436.

8) W. Bauhofer and J. Z. Kovacs: Compos. Sci. Technol. 69 (2009) 14861498.

9) T. Takeda, Y. Shindo, Y. Kuronuma and F. Narita: Polymer 52 (2011) 3852-3856.

10) T. Takeda, F. Narita, Y. Kuronuma and Y. Shindo: Int. J. Mater. Struct. Integr., in press.

11) C. Li, E. T. Thostenson and T.-W. Chou: Compos. Sci. Technol. 68 (2008) 1227-1249.

12) Y. Shindo, Y. Kuronuma, T. Takeda, F. Narita and S.-Y. Fu: Compos. Part B 43 (2012) 39-43.

13) S. M. Cardoso, V. B. Chalivendra, A. Shukla and S. Yang: Eng. Fract. Mech. 96 (2012) 380-391.

14) C. Li, E. T. Thostenson and T.-W. Chou: Compos. Sci. Technol. 68 (2008) 1445-1452.

15) G. C. Sih: Int. J. Fract. 10 (1974) 305-321.

16) W. K. Wilson: Methods of Analysis and Solutions of Crack Problems, ed. by G. C. Sih, (Noordhoff International Publishing, Leyden, The Netherlands, 1973) pp. 484-515.

17) T. Takeda, Y. Shindo, F. Narita and Y. Mito: Mater. Trans. 50 (2009) $436-445$. 